\title{
Amplitude Noise of Femtosecond Fiber Lasers in Different Modelocking Regimes
}

\author{
L. Budunoglu, F. Ö. Ilday \\ Physics Department, Bilkent University, Ankara 06800, Turkey \\ Tel: 90-312-290-1076, Fax: 90-312-266-4365,Email: budun@fen.bilkent.edu.tr
}

\begin{abstract}
Amplitude noise of femtosecond fiber laser oscillators are characterized in soliton, similariton, stretched-pulse and all-normal GVD regimes of mode-locking. Integrated relative intensity noise as low as $0.023 \%(1 \mathrm{~Hz}-100 \mathrm{kHz})$ is reported. (C)2007 Optical Society of America

OCIS codes: (320.7090) Ultrafast lasers; (140.3510) Lasers, fiber; (060.7140) Ultrafast processes in fibers; (140.4050) Mode-locked lasers
\end{abstract}

Femtosecond fiber lasers have been attracting much attention in the recent years, primarily due to their significant practical advantages over bulk solid-state lasers, including greater stability and freedom from misalignment, compact design, and relative ease of construction with inexpensive components. While fiber laser oscillators offered modest pulse energies until a few years ago, recent progress has put them in the same league as most bulk solid state lasers in terms of pulse energy and peak power. Furthermore, they are finding increasingly common usage as seed oscillators for fiber amplifier chains as well as traditional solid state amplifiers. In parallel, fiber oscillators are increasing important in optical frequency metrology applications. In virtually all applications, laser noise is one of the crucial characteristics of any laser system. Amplitude noise directly influences signal to noise ratio in most experiments. The significance of noise is even greater when the oscillator is used to seed an amplifier, in metrology applications or in experiments looking for weak signals.

However, there is a very limited number of studies characterizing the noise processes in ultrafast fiber lasers. Significantly, to the best of our knowledge, there is no systematic study of the noise characteristics of fiber oscillators operating various mode-locking regimes, despite the rich variety of pulse-shaping schemes compatible with femtosecond pulse formation in fiber oscillators. In this study, we provide amplitude noise (relative intensity noise, RIN) of Yb-doped fiber oscillators in soliton [1], stretched-pulse (dispersion-managed soliton) [2], similariton [3] and all-normal dispersion [4] regimes.

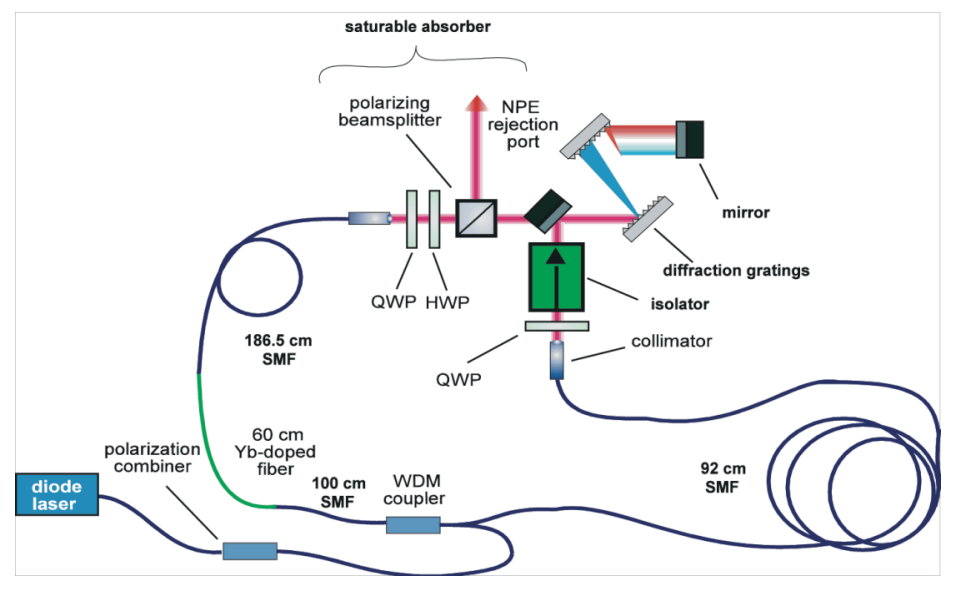

Fig. 1. Schematic of the Yb-doped fiber laser used in the experiments.

A Yb-doped fiber oscillator is preferred due to the possibility of conveniently switching from one regime to another simply by changing the amount of anomalous dispersion imparted by the intra-cavity diffraction grating compressor, or completely removing it in the case of all-normal dispersion operation. A unidirectional ring cavity increases the likelihood of self-starting operation [5]. Highly-doped (23,900 ppm) $\mathrm{Yb}$ gain fiber allows the use of a short $(\sim 60 \mathrm{~cm})$ gain segment, which follows a $\sim 1.9-\mathrm{m}$ segment of single- 


\section{ThA3.pdf}

mode fiber (SMF). Following the gain fiber, the pulse passes through a $10 \%$ output coupler with total lead fibers of $\sim 1.9-\mathrm{m}$ and then into an open-air section. Saturable absorber action is achieved through nonlinear polarization rotation [6], implemented with manual mechanical polarization controllers and a polarizing beamsplitter cube. A pair of diffraction gratings provide anomalous GVD, before the beam pass through a bulk isolator and returning to the SMF. The laser is pumped in-core by a $980-\mathrm{nm}$, SMF-coupled pump diodes, providing maximum pump power of $\sim 500 \mathrm{~mW}$. The output for the noise measurements is taken from the polarization rejection port, since this is port that provides maximum pulse energy and is commonly used in applications. The pulse repetition rate was $40 \mathrm{MHz}$ in the experiments reported here.

Total Dispersion: $-0.071 \mathrm{ps}^{2}$

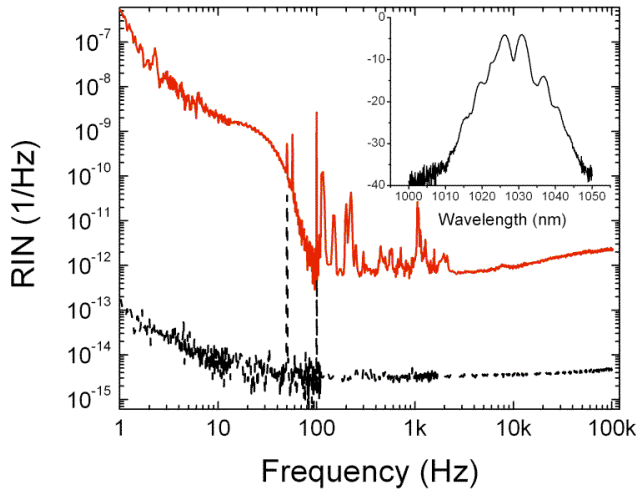

Total Dispersion: $0.011 \mathrm{ps}^{2}$

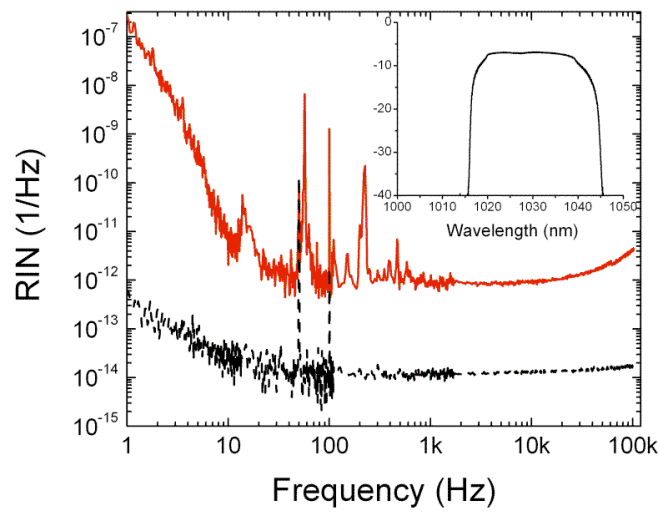

Total Dispersion: $0.005 \mathrm{ps}^{2}$

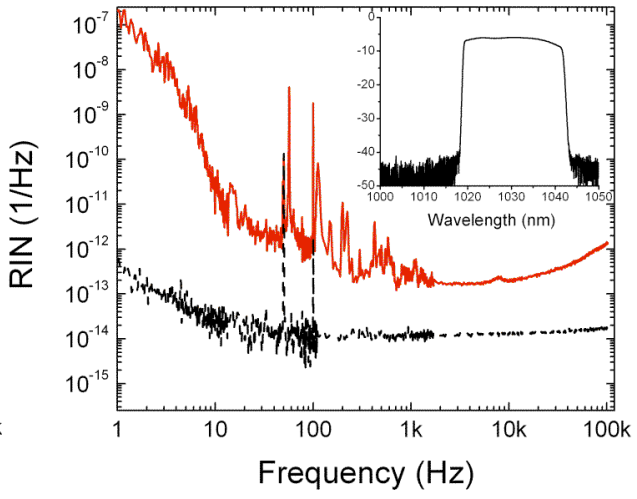

Total Dispersion: $0.020 \mathrm{ps}^{2}$

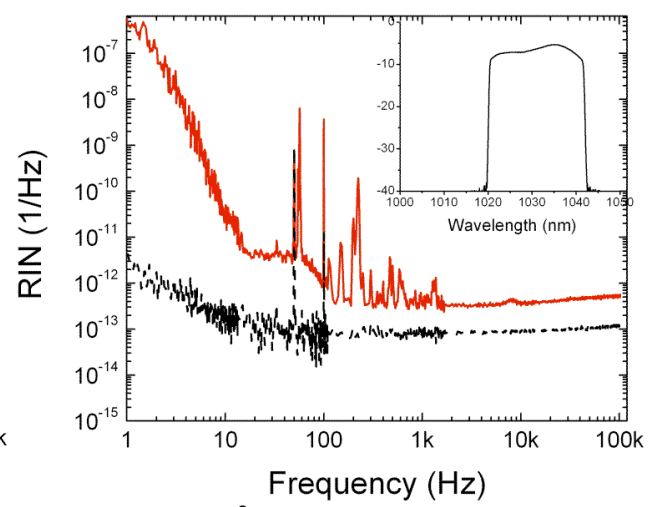

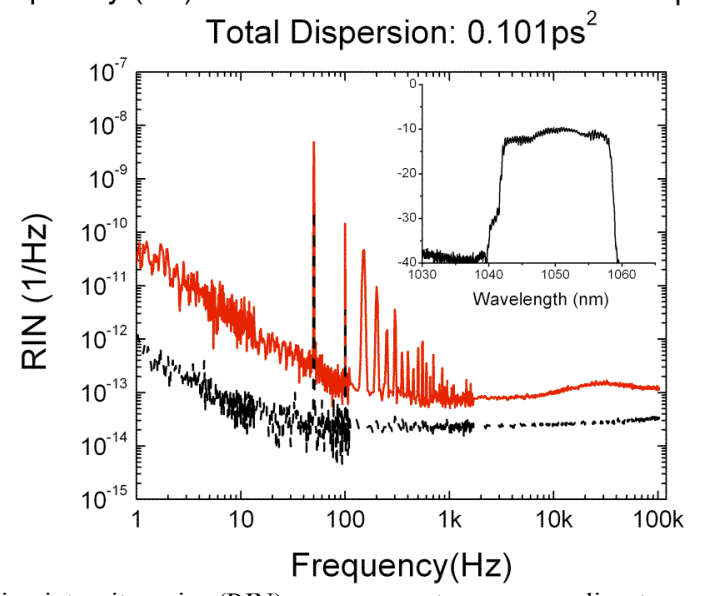

Fig. 2. Normalized relative intensity noise (RIN) measurements, corresponding to various mode-locking regimes. Insets show the corresponding optical spectrum in logarithimic scale as obtained from the $10 \%$ output coupler. The black, dashed lines indicate the noise floor of the measurement setup.

The amplitude noise of the lasers were characterized with a dynamic signal analyzer (Stanford Research Systems, SR785), following photodetection with a free-space InGaAs detector, filtering off frequencies 


\section{ThA3.pdf}

higher than $1 \mathrm{MHz}$ and subsequent amplification with a low-noise preamplifier (Stanford Research Systems, SR560). The high-frequency limit to the measurements is $102 \mathrm{kHz}$, imposed on by the dynamic signal analyzer. Since the goal was to characterize the relative effect of the various pulse-shaping regimes on the noise performance, no particular effort was made to minimize excess environmental noise: In fact, the laser setup was constructed on an optical breadboard, which is not enclosed in a box (which is known to reduce low-frequency noise significantly), the pump diode is driven at constant current, instead of constant optical power, and the measurements were taken while a noisy large computer mainframe and airconditioning system were operational in the room. Despite these adverse conditions, the overall noise level of the quieter oscillator configurations were at the same level as some of the lowest-noise femtosecond fiber lasers reported before (the integrated RIN of the Er-doped fiber laser was measured to be $0.03 \% \mathrm{rms}$ from $10 \mathrm{kHz}$ to $1 \mathrm{MHz}$ in Ref. [7]). Amplitude noise measurements obtained at various net cavity dispersion settings and correspoding to soliton-like, stretched-pulse (near-zero dispersion), stretched-pulse (large normal dispersion), similariton-like and all-normal dispersion, respectively, are shown in Fig. 2. All measurements are after the noise level was minimized through adjusting the polarization settings, while observing the noise level in real time. The noise floor levels are normalized by the same voltage calibration factor as the individual noise measurements, only to indicate the noise floor. The key parameters of the measurements are summarized in Table 1.

\begin{tabular}{|c|c|c|c|c|}
\hline $\begin{array}{c}\text { Net Cavity } \\
\left.\text { Dispersion } \mathbf{( p s}^{\mathbf{2}}\right)\end{array}$ & $\begin{array}{c}\text { Optical Bandwidth } \\
\mathbf{( n m})\end{array}$ & $\begin{array}{c}\text { Optical Power } \\
\mathbf{( m W )}\end{array}$ & $\begin{array}{c}\text { Total Noise } \\
(\mathbf{1 ~ H z}-\mathbf{1 0 0} \mathbf{~ k H z})\end{array}$ & $\begin{array}{c}\text { High Frequency } \\
\text { Noise (1-100 kHz) }\end{array}$ \\
\hline-0.071 & 8 & 40 & $0.13 \%$ & $0.084 \%$ \\
\hline+0.005 & 23 & 25 & $0.088 \%$ & $0.050 \%$ \\
\hline+0.011 & 22 & 22 & $0.11 \%$ & $0.092 \%$ \\
\hline+0.020 & 20 & 40 & $0.12 \%$ & $0.043 \%$ \\
\hline+0.101 & 15 & 42 & $0.025 \%$ & $0.023 \%$ \\
\hline
\end{tabular}

Table 1. Experimental measurements corresponding to various mode-locking regimes: soliton, stretched-pulse (near zero dispersion), stretched-pulse (normal dispersion), similariton, all-normal dispersion.

In conclusion, we have characterized the amplitude noise in femtosecond fiber lasers, operating in various mode-locking regimes and over key laser parameters such as optical power. The measurements indicate that the operational characteristics are more important in determining laser noise than the regime of modelocking. Changing the polarization settings by a small amount, such that the spectrum is virtually unchanged, can easily increase the laser noise several factors. It appears that, at the present time, there is no simple indicator to know that the noise of the laser has been minimized, without actually carrying out a measurement. High-frequency $(>1 \mathrm{kHz})$ is the more significant noise characteristic, since noise at the lower frequencies is determined primarily by the environmental factors, thus, varying from measurement to measurement. There is preliminary evidence that high-frequency noise tends to decrease with increasing net normal (positive) cavity dispersion, which is not surprising considering that these regimes corresponding to milder pulse-shaping due to minimization of soliton-like processes (negative chirp + Kerr nonlinearity). However, further investigations are needed before this trend can be confirmed. Finally, even the measurements of relatively high noise correspond to fairly low fluctuations. Thus, these measurements confirm that fiber lasers can be a reliable source of femtosecond pulses with very low amplitude fluctuations, even for configurations that are not optimized for noise.

\section{References:}

[1] I. N. Duling III, "Subpicosecond all-fiber erbium laser," Electron. Lett. 27, 544 (1991).

[2] K. Tamura, J. Jacobson, H.A. Haus, E.P. Ippen, and J.G. Fujimoto, "77-fs pulse generation from a stretched-pulse modelocked all-fiber ring laser," Opt. Lett. 18, 1080 (1993).

[3] F. O. Ilday, J. R. Buckley, W. G. Clark, and F. W. Wise, "Self-Similar Evolution of Parabolic Pulses in a Laser," Phys. Rev. Lett. 92, 3902 (2004).

[4] A. Chong, J. Buckley, W. Renninger, and F. W. Wise, “All-normal-dispersion femtosecond fiber laser,” Opt. Exp. 14, 10095 (2006).

[5] K. Tamura, J. Jacobson, E. P. Ippen, H. A. Haus, and J. G. Fujimoto, “Unidirectional ring resonators for self-starting passively mode-locked lasers" Opt. Lett. 18, 220 (1993).

[6] M. Hofer, M. E. Fermann, F. Harberl, M. H. Ober, and A. J. Schmidt, "Mode locking with cross-phase and self-phase modulation," Opt. Lett. 16, 502 (1991).

[7] F. Ö. Ilday, J. Chen, A. Winter, F. X. Kaertner, F. W. Wise, O. Shkurikhin, and D. Gapontsev, "Low-noise, high-energy, singlemode, femtosecond fiber laser system," Proceedings of Conference on Lasers and Electro-Optics, Baltimore, MA, 2005. 